\title{
A paleo Tibet-Myanmar connection? Reconstructing the Late Eocene drainage system of central Myanmar using a multi-proxy approach Supplementary Data
}

A. Licht, C. France-Lanord, L. Reisberg, C. Fontaine, Aung Naing Soe and J.J. Jaeger

\section{Annexe 1 : Dataset}

All samples were collected during the 2011 and 2012 expeditions of the French-Myanmar palaeontological team; nearest village, GPS coordinates, dominant lithology and analytical techniques employed are given in table A1. All samples were selected in a $25 \mathrm{~km}$ wide area around Bahin township (area A in figure 1A). A precise geological map of the area is available in Aung Naing Soe et al. (2002).

\begin{tabular}{|c|c|c|c|c|c|}
\hline Sample & Village Locality & Sampling site & $\begin{array}{l}\text { Dominant } \\
\text { lithology }\end{array}$ & Formation & Analyses \\
\hline GA & Ganle & $\begin{array}{l}\mathrm{N} 21^{\circ} 44^{\prime} 03.0^{\prime \prime} \\
\mathrm{E} 094^{\circ} 43^{\prime} 26.3^{\prime \prime}\end{array}$ & Mudstones & Pondaung & $\begin{array}{l}\mathrm{Nd} \text { and } \mathrm{Sr} \text { isotopic analysis on the }<2 \mu \mathrm{m} \text { and } \\
>63 \mu \mathrm{m} \text { fraction, DRX on the }<2 \mu \mathrm{m} \text { fraction }\end{array}$ \\
\hline GAN-08 & Ganle & $"$ & Mudstones & $"$ & $\mathrm{Nd}$ and $\mathrm{Sr}$ isotopic bulk analysis \\
\hline PA & Pangan & $\begin{array}{l}\mathrm{N} 21^{\circ} 42^{\prime} 31.0^{\prime \prime} \\
\mathrm{E} 094^{\circ} 49^{\prime} 21.6^{\prime \prime}\end{array}$ & sandstones & $"$ & $\begin{array}{l}\mathrm{Nd} \text { and } \mathrm{Sr} \text { isotopic analysis on the }<2 \mu \mathrm{m} \text { and } \\
>63 \mu \mathrm{m} \text { fraction, DRX on the }<2 \mu \mathrm{m} \text { fraction }\end{array}$ \\
\hline PK1-03 & Paukkaung & $\begin{array}{l}\mathrm{N} 21^{\circ} 45^{\prime} 10.3^{\prime \prime} \\
\mathrm{E} 094^{\circ} 38^{\prime} 12.0^{\prime \prime}\end{array}$ & sandstones & $"$ & QFL point counting, heavy minerals counting \\
\hline PK2-06 & Paukkaung & $\begin{array}{l}\text { N21 } 1^{\circ} 45^{\prime} 16.3^{\prime \prime} \\
\text { E094 } \\
\end{array}$ & sandstones & " & $\begin{array}{l}\text { Nd and } \mathrm{Sr} \text { isotopic bulk analysis, QFL point } \\
\text { counting, heaw minerals counting }\end{array}$ \\
\hline PK2-17 & Paukkaung & $"$ & sandstones & $"$ & QFL point counting, heavy minerals counting \\
\hline PK2-21 & Paukkaung & $"$ & sandstones & " & $\begin{array}{l}\mathrm{Nd} \text { and } \mathrm{Sr} \text { isotopic bulk analysis, QFL point } \\
\text { counting, heawy minerals counting }\end{array}$ \\
\hline $\mathrm{TH}$ & Thaminchauk & $\begin{array}{l}\text { N21 } 1^{\circ} 45^{\prime} 41.0^{\prime \prime} \\
\text { E094 } \\
\end{array}$ & Siltstones & $"$ & $\begin{array}{l}\mathrm{Nd} \text { and } \mathrm{Sr} \text { isotopic analysis on the }<2 \mu \mathrm{m} \text { and } \\
>63 \mu \mathrm{m} \text { fraction, DRX on the }<2 \mu \mathrm{m} \text { fraction }\end{array}$ \\
\hline THT1 & Thaminchauk & $"$ & Mudstones & $"$ & DRX \\
\hline $\mathrm{P} 1$ to $\mathrm{P} 2$ & Than U Daw & $\begin{array}{l}\text { N2 } 21^{\circ} 41^{\prime} 07.4^{\prime \prime} \\
\text { E094 } 48^{\prime} 37.5^{\prime \prime}\end{array}$ & Pebbles & $"$ & Thin section, QFL point counting for P12 \\
\hline YAS-06 & Yarshe & $\begin{array}{l}\text { N21 } 1^{\circ} 44^{\prime} 12.5^{\prime \prime} \\
\text { E094 } 38^{\prime} 15.3^{\prime \prime}\end{array}$ & Mudstones & $"$ & $\mathrm{Nd}$ and $\mathrm{Sr}$ isotopic bulk analysis \\
\hline YAS-21 & Yarshe & " & Mudstones & $"$ & DRX \\
\hline YPL-11 & Nyaungpinle & $\begin{array}{l}\mathrm{N} 21^{\circ} 45^{\prime} 03.8^{\prime \prime} \\
\mathrm{E} 094^{\circ} 37^{\prime} 35.3^{\prime \prime}\end{array}$ & sandstones & $"$ & $\begin{array}{l}\mathrm{Nd} \text { and } \mathrm{Sr} \text { isotopic bulk analysis, heavy minerals } \\
\text { counting }\end{array}$ \\
\hline YAW2 & Seikche North & $\begin{array}{l}\text { N21 } 1^{\circ} 42^{\prime} 20.4^{\prime \prime} \\
\text { E094 } 42^{\prime} 41.6^{\prime \prime}\end{array}$ & sandstones & Yaw & Heavy minerals counting \\
\hline YAW-SABLE & Seikche North & $"$ & sandstones & $"$ & $\begin{array}{l}\mathrm{Nd} \text { and } \mathrm{Sr} \text { isotopic analysis on the }<2 \mu \mathrm{m} \text { and } \\
>63 \mu \mathrm{m} \text { fraction, DRX on the }<2 \mu \mathrm{m} \text { fraction }\end{array}$ \\
\hline YAW-A & Seikche South & $\begin{array}{l}\mathrm{N} 21^{\circ} 42^{\prime} 40.5^{\prime \prime} \\
\mathrm{E} 094^{\circ} 42^{\prime} 57.0^{\prime \prime}\end{array}$ & Mudstones & $"$ & $\mathrm{Nd}$ and $\mathrm{Sr}$ isotopic bulk analysis \\
\hline YAWS & Seikche South & $"$ & sandstones & $"$ & $\begin{array}{l}\text { Nd and } \mathrm{Sr} \text { isotopic bulk analysis, QFL point } \\
\text { counting }\end{array}$ \\
\hline YAW-RE & Bahin & $\begin{array}{l}\mathrm{N} 21^{\circ} 43^{\prime} 32.6^{\prime \prime} \\
\mathrm{E} 094^{\circ} 40^{\prime} 39.7^{\prime \prime}\end{array}$ & Mudstones & $"$ & $\mathrm{Nd}$ and $\mathrm{Sr}$ isotopic bulk analysis \\
\hline YTP & Bahin & $\begin{array}{l}\text { N21 }{ }^{\circ} 43^{\prime} 11.1^{\prime \prime}, \\
\text { E094 } 40^{\prime} 23.8^{\prime \prime}\end{array}$ & Siltstones & $"$ & $\mathrm{Nd}$ and $\mathrm{Sr}$ isotopic bulk analysis \\
\hline
\end{tabular}

Table A1: Studied samples 


\section{Annexe 2: Palaeocurrent data}

Palaeocurrent data were acquired in two distinct areas : in the Bahin township, where the Pondaung Formation crops out extensively (area $\mathrm{A}$ in figure 1A; precise locations in annexe 1), and in the Kalewa township, where the Yaw Formation crops out (area B in figure 1A; exposure sites along the Kalewa-Kalaymyo road, $23^{\circ} 12^{\prime} 48^{\prime \prime} \mathrm{N} 94^{\circ} 19^{\prime} 06^{\prime \prime} \mathrm{E}$ to $23^{\circ} 11^{\prime} \mathrm{N} 94^{\circ} 3^{\prime} \mathrm{E}$ ). Data are summarized in table A2. Paleoflow directions were measured on the set axes of trough cross-beddings on 3D exposures. Selected sets are sufficiently large $(>40 \mathrm{~cm})$ to correspond to the dominant flow direction (Collinson and Thompson, 1989). The structural dip at the selected sites was always below $25^{\circ}$, which is sufficiently small to avoid any tectonic correction (Collinson and Thompson, 1989).

\begin{tabular}{|c|c|c|c|c|}
\hline Rock Unit & Location & directional indicators & Data & $\begin{array}{l}\text { Mean paleoflow } \\
\text { direction }\end{array}$ \\
\hline Pondaung Fm & $\begin{array}{c}\text { Minbu Sub-Basin, Myaing } \\
\text { District }\end{array}$ & $\begin{array}{l}\text { Trough cross bedding (facies St } \\
\text { of Aung Naing Soe et al., 2002) }\end{array}$ & $\begin{array}{l}278 \text { crossbeds on } 66 \\
\text { distinct channel bodies }\end{array}$ & $243^{\circ}$ \\
\hline Yaw Fm & $\begin{array}{l}\text { Chindwin Sub-Basin, } \\
\text { Kalewa township }\end{array}$ & $\begin{array}{c}\text { Trough cross bedding (facies } \\
\text { St, this paper) }\end{array}$ & $\begin{array}{l}171 \text { crossbeds on } 37 \\
\text { distinct channel bodies }\end{array}$ & $257^{\circ}$ \\
\hline
\end{tabular}

Table A2:Palaeocurrent data

\section{Annexe 3 : Petrographic analysis}

Detailed results of the point-counting analyses are given in table A3; results of the heavy mineral counting are presented in the main document. More samples were initially prepared for heavymineral identification, but the very low abundances of heavy minerals in several samples did not allow us to study the results.

\begin{tabular}{|l|r|r|r|r|r|r|r|}
\hline & Q & F & L & Lv & Ls & Lm & \multicolumn{1}{c|}{ MI } \\
\hline PK1-03 & 16 & 13 & 71 & 29 & 15 & 27 & 137 \\
\hline PK2-06 & 15 & 18 & 67 & 28 & 18 & 21 & 124 \\
\hline PK2-17 & 13 & 17 & 70 & 32 & 17 & 20 & 130 \\
\hline PK2-21 & 19 & 16 & 65 & 32 & 18 & 16 & 115 \\
\hline YAW-S & 23 & 16 & 61 & 28 & 16 & 17 & 143 \\
\hline
\end{tabular}

Table A3: results of the point-counting analyses. Q: Quartz, F: Feldspars, L: Lithics, Lv: volcanic lithics, Ls: sedimentary lithics, Lm: metamorphic lithics, MI: Metamorphic Index (Garzanti and Vezzoli, 2003). Chert lithics and ultramafic lithics were not detected. YAW-S delivered numerous carbonate lithics (gastropods and bivalve fragments), which are considered as intrabasinal and have not been counted. 


\section{$\underline{\text { Annexe 4: isotopic results }}$}

Powdered sediments were analyzed for $\mathrm{Sr}, \mathrm{Rb}, \mathrm{Sm}$ and $\mathrm{Nd}$ concentrations using a Thermo Elemental IRIS ICP-OES spectrometer at the Service d'Analyse des Roches et des Minéraux (CRPG, Vandoeuvre les Nancy - France). Nd and Sr chemical separation for isotopic measurements and mass spectrometric analyses were performed at the CRPG, according to the standard procedures of the laboratory. Briefly, after decarbonation, the silicate fractions were dissolved in $\mathrm{HF}-\mathrm{HNO}_{4}$ and a small amount of $\mathrm{HClO}_{4}$. Sr and Nd were separated using Eichrom Sr-spec, TRU-spec and Lnspec resins, following procedures closely adapted from those of Pin et al. (1994). Sr isotopic composition was measured by thermal ionization mass spectrometry, using a Triton Plus instrument operated in static mode. The ${ }^{87} \mathrm{Sr} /{ }^{86} \mathrm{Sr}$ ratios were corrected for mass fractionation assuming ${ }^{86} \mathrm{Sr} /{ }^{88} \mathrm{Sr}$ $=0.1194$. During these measurements the ${ }^{87} \mathrm{Sr} /{ }^{86} \mathrm{Sr}$ value for the NBS 987 standard was $0.710263 \pm$ $0.000023(2 \sigma) . \mathrm{Nd}$ isotopic compositions were measured using a Neptune Plus MC-ICP-MS. Nd isotopic ratios are normalized to ${ }^{146} \mathrm{Nd} /{ }^{144} \mathrm{Nd}=0.7219$. During the period of sample measurement the $\mathrm{JNdi} \mathrm{Nd}$ standard yielded a mean value of ${ }^{143} \mathrm{Nd} /{ }^{144} \mathrm{Nd}=0.512077 \pm 0.000017(2 \sigma)$. Detailed results are given in table $\mathrm{A} 4 \mathrm{Nd}$ and $\mathrm{Sr}$ procedural blanks were insignificant compared to the amount of $\mathrm{Sr}$ and $\mathrm{Nd}$ measured in the samples.

\begin{tabular}{|c|c|c|c|c|c|c|c|c|c|c|}
\hline Sample & Grain size & $\begin{array}{c}\text { Sm } \\
\text { (ppm) }\end{array}$ & $\begin{array}{c}\mathbf{N d} \\
(\mathbf{p p m})\end{array}$ & $\begin{array}{c}\mathbf{R b} \\
(\mathbf{p p m})\end{array}$ & $\begin{array}{c}\text { Sr } \\
\text { (ppm) }\end{array}$ & $87 \mathrm{Sr} / 86 \mathrm{Sr}$ & $\begin{array}{l}\text { Standard } \\
\text { error }(2 \sigma)\end{array}$ & $143 \mathrm{Nd} / 144 \mathrm{Nd}$ & $\begin{array}{l}\text { Standard } \\
\text { error }(2 \sigma)\end{array}$ & $\varepsilon \mathrm{Nd}$ \\
\hline TH (<2 $\mu$ m fraction) & Mudstones & 3.58 & 17.53 & 60.3 & 111.5 & 0.714727 & 0.000013 & 0.512263 & 0.000021 & $-7,32$ \\
\hline $\mathrm{TH}(>63 \mu \mathrm{m}$ fraction $)$ & Sandstones & 5.3 & 26.06 & 84.23 & 103.4 & 0.715563 & 0.000005 & 0.512237 & 0.000003 & $-7,82$ \\
\hline PA $(<2 \mu$ m fraction $)$ & Mudstones & 3.86 & 15.61 & 7.85 & 369.5 & 0.705455 & 0.000006 & 0.512651 & 0.000007 & 0,26 \\
\hline PA $(>63 \mu \mathrm{m}$ fraction $)$ & Sandstones & 2.69 & 11.81 & 20.56 & 888.1 & 0.705741 & 0.000010 & 0.512442 & 0.000005 & $-3,83$ \\
\hline GA (<2 $\mu$ m fraction) & Mudstones & 7.3 & 39.88 & 95.9 & 142.8 & 0.717205 & 0.000010 & 0.512438 & 0.000004 & $-3,9$ \\
\hline GA (>63um fraction) & Sandstones & 4.58 & 21.89 & 42.54 & 82.94 & 0.714055 & 0.000012 & 0.512342 & 0.000005 & $-5,78$ \\
\hline GAN08 & Mudstones & 4.22 & 22.09 & 98.07 & 118.9 & 0.717786 & 0.000014 & 0.512319 & 0.000005 & $-6,23$ \\
\hline PK2-06 & Sandstones & 4.32 & 20.48 & 46.71 & 256.9 & 0.705905 & 0.000008 & 0.512627 & 0.000006 & -0.21 \\
\hline PK2-21 & Sandstones & 4.12 & 19.41 & 55.18 & 96.05 & 0.708842 & 0.000009 & 0.512521 & 0.000005 & -2.28 \\
\hline YAS-06 & Mudstones & 4.58 & 21.51 & 84.99 & 119 & 0.715201 & 0.000014 & 0.512240 & 0.000004 & -7.76 \\
\hline YPL-11 & Sandstones & 9.59 & 47.32 & 68.41 & 96.05 & 0.711181 & 0.000020 & 0.512553 & 0.000006 & -1.66 \\
\hline YAWS & Sandstones & 8.43 & 35.84 & 59.73 & 173.5 & 0.711771 & 0.000015 & 0.512254 & 0.000003 & $-7,49$ \\
\hline YS $(<2 \mu m$ fraction $)$ & Mudstones & 4.91 & 24.34 & 76.14 & 113.6 & 0.712084 & 0.000013 & 0.512511 & 0.000004 & $-2,48$ \\
\hline 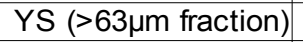 & Sandstones & 3.63 & 16.31 & 42.34 & 108.5 & 0.709754 & 0.000014 & 0.512371 & 0.000020 & $-5,2$ \\
\hline YTP & Siltstones & 5.06 & 23.09 & 37.93 & 269.7 & 0.708533 & 0.000011 & 0.512363 & 0.000009 & -5.36 \\
\hline YAW-A & Mudstones & 5.27 & 24.72 & 99.38 & 101.3 & 0.713161 & 0.000008 & 0.512268 & 0.000013 & $-7,22$ \\
\hline YAW-RE & Mudstones & 4.68 & 22.91 & 97.25 & 129.5 & 0.713984 & 0.000021 & 0.512294 & 0.000007 & -6.71 \\
\hline
\end{tabular}

Table A4: Sm, Nd, Rb and Sr contents, ${ }^{87} \mathrm{Sr}{ }^{86} \mathrm{Sr}$ and ${ }^{143} \mathrm{Nd} /{ }^{144} \mathrm{Nd}$ ratios from thirteen samples of the Pondaung and Yaw formations. For four samples results for both fine $(<2 \mu \mathrm{m})$ and coarse $(>63 \mu \mathrm{m})$ fractions are shown. For the remaining samples, only bulk analyses were performed. 